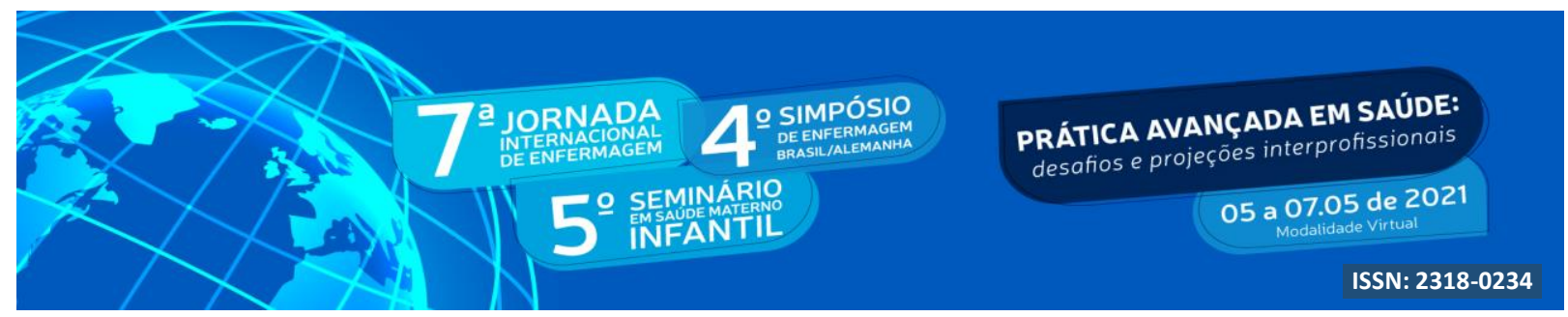

DOI: http://doi.org/10.48195/jie2021-010

\title{
TECNOLOGIA, REDES SOCIAIS E A RELAÇÃO PROFISSIONAL DA SAÚDE/PACIENTE ${ }^{1}$
}

\author{
Adriani Cheron Vielmo²; Caroline Bortolas de Carvalho ${ }^{3}$; Letícia Westphalen Bento ${ }^{4}$
}

\begin{abstract}
RESUMO
O objetivo do estudo foi realizar uma revisão narrativa da literatura dos aspectos bioéticos envolvidos no uso das tecnologias, redes sociais na relação médico/paciente (RMP), utilizando os descritores: bioética, rede social, confidencialidade e autonomia nas seguintes bases de dados: Scielo, PubMed e Google Scholar. Verificou-se que apesar do crescimento constante do uso das tecnologias e redes sociais pelos profissionais da área da saúde, ainda é escasso os estudos que pautam sobre a privacidade, confidencialidade e bioética nesse contexto. Dessa forma, salienta-se ser de extrema importância o desenvolvimento de reflexões relativas aos princípios bioéticos do uso de rede sociais os profissionais da saúde e seus pacientes. Os aspectos bioéticos do uso de informações dos pacientes e sua divulgação deve ser amplamente discutido entre profissionais da área da saúde no intuito de garantir o sigilo dos dados por eles acessados, a privacidade dos pacientes e a qualidade das informações disponibilizadas ao publico geral.
\end{abstract}

Palavras-chave: Bioética; Rede social; Confidencialidade; Autonomia.

\begin{abstract}
The objective of the study was to carry out a narrative review of the literature on the bioethical aspects involved in the use of technologies, social networks in the doctor / patient relationship (RMP), using the descriptors: bioethics, social network, confidentiality and autonomy in the following databases: Scielo , PubMed and Google Scholar. It was found that despite the steady growth in the use of technologies and social networks by health professionals, there are still few studies on privacy, confidentiality and bioethics in this context. Thus, it is emphasized that it is extremely important to develop reflections on the bioethical principles of the use of social networks for health professionals and their patients. The bioethical aspects of the use of patient information and its dissemination must be widely discussed among health professionals in order to guarantee the confidentiality of the data they access, the privacy of the patients and the quality of the information made available to the general public.
\end{abstract}

Key Words: Bioethics; Social network; Confidentiality; Autonomy.

\footnotetext{
${ }^{2}$ Estudante do Mestrado em Ciências da Saúde e da Vida. Universidade Franciscana -UFN. E-mail: adricheron@hotmail.com

${ }^{3}$ Estudante do Mestrado em Ciências da Saúde e da Vida. Universidade Franciscana -UFN. E-mail: carolbortolas@yahoo.com.br

${ }^{4}$ Orientador. Doutorado em Odontologia. Universidade Franciscana. E-mail: leticiabento@ufn.edu.br
} 


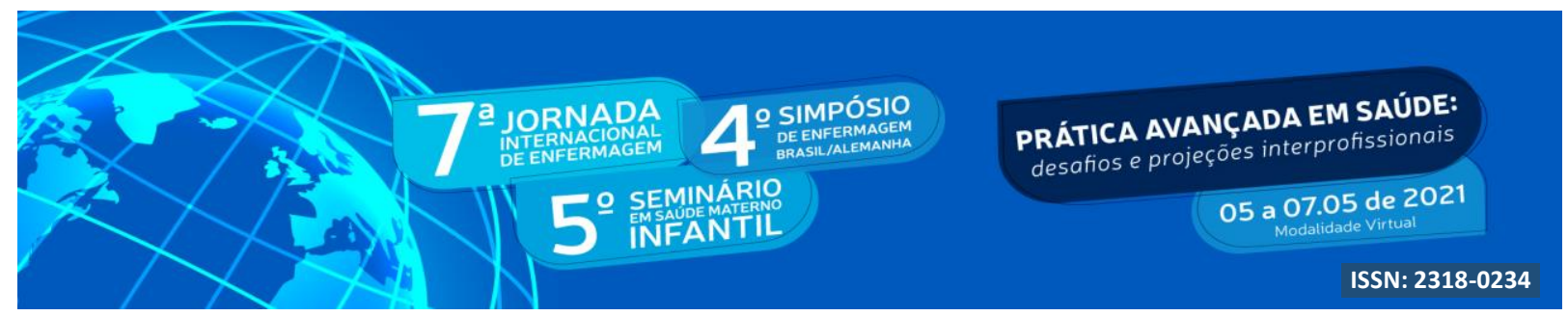

\section{INTRODUÇÃ̃o}

O aumento do uso das redes sociais vem crescendo no Brasil. São pelo menos oitenta milhões de brasileiros com contas ativas em Social Networking Sites como Facebook, Instagram e Twitter. Essa nova prática tecnológica tem dado um novo aspecto para um clássico dilema da relação profissional/paciente, como a violação do direito ao sigilo, à privacidade e à confidencialidade da informação. Temos que destacar que a preservação de informações é um dever prima facie de qualquer profissional de saúde que estabeleça um vínculo com um paciente (NASCIMENTO, 2016; POSSOLLI; DO NASCIMENTO; DA SILVA, 2015).

A bioética analisou inicialmente os conflitos relacionados à complexa densidade tecnológica intrínseca ao setor saúde. As mudanças da relação profissional da saúde-paciente juntamente com o uso de tecnologias externas está cada vez mais em evidencia na vida dos profissionais da área, porém somadas aos progressos tecnológicos que impactam a rotina das pessoas tem feito emergir situações em que informações de pacientes são expostas publicamente têm ficado mais constante e os conselhos profissionais têm o papel de fiscalizar o comportamento ético de seus inscritos (MARTORELL, 2017). Apesar disso, é esperado uma conduta responsável e eticamente aceitável daqueles que tem acesso a informações particulares. $\mathrm{Na}$ área da saúde, considerando as informações acessadas pelos profissionais durante a prática de suas funções, vem a tona o pensamento Hipocrático: "Tudo quanto veja ou ouça, profissional ou privadamente, que se refira à intimidade humana e não deva ser divulgado, eu manterei em segredo e contarei a ninguém". Este compromisso, frequentemente juramentado em solenidades públicas de colação de grau de cursos da área da saúde, também é algo esperado por parte dos pacientes (MARTORELL; NASCIMENTO; GARRAFA, 2015; SALES-PERES et al., 2008).

O campo da bioética traz reflexões importantes sobre os desafios éticos encontrados na área da saúde. Explica também as questões relacionadas à autonomia do paciente valorizando a comunicação necessária às boas práticas, permitindo assim ao profissional entender que sua ação pode envolver conflitos de ordem moral, exigindo ponderação entre recursos e a 


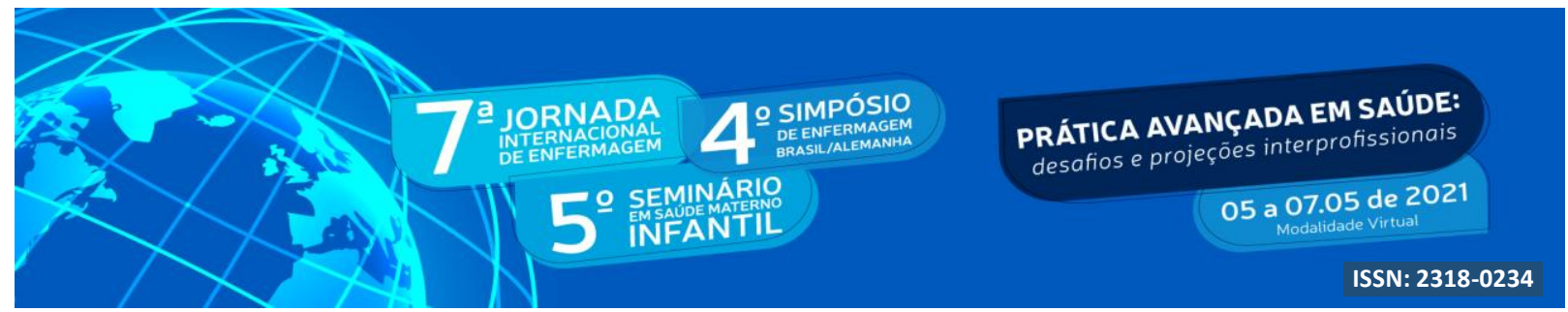

condição orgânica do paciente. Essas discussões contribuem para aprimorar a assistência e as habilidades interpessoais de profissionais de saúde, paciente e família, sendo extremamente importante considera-la para tomar decisões prudentes (CAMPOS; SILVA; SILVA, 2019; LIMA; BIASOLI, 2018).

O uso das mídias sociais na área da saúde traz à tona conflitos morais que antes não eram experimentados. Encontra-se na bioética princípios que podem norterar o uso adeuqado desta tecnologia que tem cada vez mais presença na vida dos profissionais da área. Os profissionais de saúde passaram a ofertar informações sobre educação em saúde a população, gerando outro meio de contato direto com seus pacientes, divulgando assim seus serviços ou ainda compartilhando informações de seus interesses com de seus colegas (FELTER et al., 2017).

As mudanças da relação profissional da saúde-paciente somadas aos progressos tecnológicos que impactam a rotina das pessoas têm feito emergir situações em que profissionais de saúde geram risco (real ou potencial) de ameaça a confidencialidade aos seus pacientes e, portanto, também urgir uma necessidade de intervenção imediata (NORMAN; AIKINS; BINKA, 2011).

\section{OBJETIVO}

O objetivo desse artigo é realizar uma revisão de literatura de alguns aspectos bioéticos envolvidos no uso de redes sociais e a relação médico/paciente (RMP).

\section{METODOLOGIA}

Trata-se de um estudo de revisão narrativa (RN) da literatura, realizada de abril de 2020 a fevereiro de 2021. Foi realizado uma busca sobre a relação equipe de saúde/paciente e o uso de tecnologias e redes sociais, utilizando os seguintes descritores no idioma adequado para cada uma das bases de dados: Bioethics; Social network; Confidentiality; Autonomy. Foram utilizadas as bases de dados: Scielo, PubMed e Google Scholar. Foram incluídos artigos em inglês e português dos últimos 10 anos que abordavam o tema de pesquisa. 


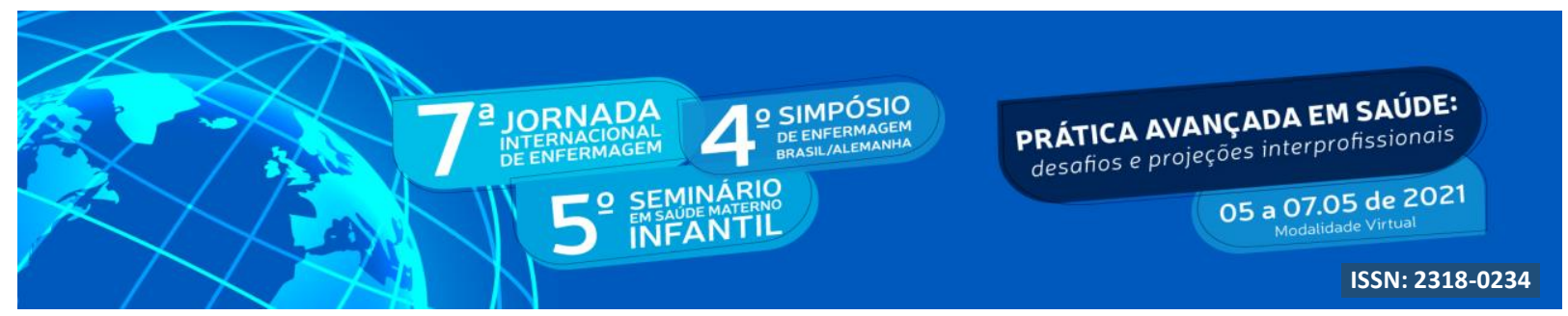

\section{RESULTADOS E DISCUSSÃO}

A tecnologia da informação é uma nova forma de dar suporte à equipe na tomada de decisão, com ferramentas de apoio à escolha clínica. Apesar dos benefícios das novas tecnologias serem diversos, também há desvantagens, incluindo o risco de perda da privacidade e confidencialidade na relação equipe-paciente, assim como a autonomia (NORMAN; AIKINS; BINKA, 2011).

De redes sociais o Facebook é o mais popular. Segundos dados da própria companhia, "uma em cada três pessoas nos Estados Unidos - mais de 128 milhões de pessoas - visitam o Facebook todos os dias, e cerca de 24 milhões no Reino Unido fazem o mesmo (LIMA; CRUZ; DA SILVA, 2016). No Brasil, o número de pessoas de possuíam um perfil no Facebook chegou aos 73 milhões, sendo um número alto, se considerarmos que, no país, existem 94 milhões de pessoas com acesso à internet (MARTORELL, 2017). Importante atualizar esses números.

Um estudo realizado por Thompson et.al. (2018), procurou identificar a frequência e o conteúdo das redes sociais entre os estudantes e residentes de medicina, tendo como objetivo pesquisar os perfis do Facebook desse público, havendo 501 estudantes de medicina e 312 residentes da Universidade da Flórida. Obteve-se como resultado, que o Facebook é uma rede social comum entre os médicos e estagiários, sendo que $44,5 \%$ possuem uma conta. Os estudantes de medicina usam a rede com uma frequência de 64,3\% e os residentes com uma frequência menor.

Um estudo realizado com 139 pacientes e 153 profissionais da área da saúde na Holanda teve como finalidade identificar o modo como utilizavam as redes sociais. A avaliação das respostas dos questionários apontou que a $43 \%$ dos profissionais da saúde utiliza a rede social Facebook, 14\% aparecendo como motivação de uso da rede: a comunicação profissional-paciente, $20 \%$ marketing, e a comunicação com colegas $22 \%$ ANTHEUNIS; TATES; NIEBOER, 2012). Já outro estudo mostrou que os profissionais de saúde têm utilizado as redes sociais para a propagação de mensagens relacionadas com a promoção da saúde, a educação médica e a divulgação de conferências (MCKEE, 2013). 


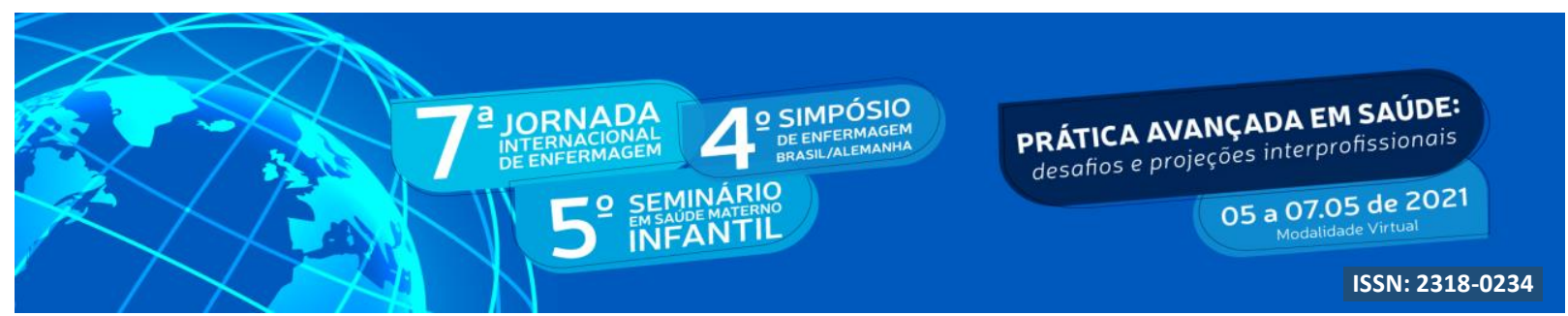

Lima, Cruz e Silva (2016), descrevem que o marketing pode ser definido como o processo social por meio do qual as pessoas obtêm aquilo que necessitam e o que desejam com a criação, oferta e livre negociação de produtos e serviços com os outros. Devido ao crescimento das redes sociais virtuais, muitos setores da economia passaram a identificar perfis do Facebook e Instagram como ferramentas para interagir com o público-alvo e realizar a publicidade e propaganda dos seus serviços e produtos. Por causa do seu alcance, as redes sociais virtuais estabeleceram certa popularidade entre os profissionais de saúde e têm sido amplamente utilizadas para propaganda. No entanto, é necessário atentar para as normas que regem o uso da publicidade e propaganda por parte de cada conselho profissional.

As transformações na relação profissional da saúde-paciente somadas aos progressos tecnológicos que influenciam a rotina das pessoas têm feito emergir situações em que profissionais da saúde geram risco real ou potencial aos seus pacientes e, desse modo, também urgir uma necessidade de intervenção imediata (MARTORELL, 2017) .

Garrafa (2009) entende que uma parte dos conflitos morais na saúde emerge de contextos apontados pela evolução da tecnologia. Para o autor, os conflitos morais em saúde podem ser ordenados em duas perspectivas: problemas persistentes e emergentes em bioética. A bioética de situações emergentes, é aquela que se ocupa "dos conflitos originados pela contradição verificada entre o progresso biomédico desenfreado dos últimos anos e os limites ou fronteiras da cidadania e dos direitos humanos, como as fecundações assistidas, as doações e transplantes de órgãos e tecidos, o engenheiramento genético de animais e da própria espécie humana e inúmeras outras situações."

Para Martorell (2017) a super exposição entre o público e o privado envolvendo o uso de redes sociais dão sinal de imaturidade dos usuários em relação ao alcance e possibilidade de uso de informações que os mesmos compartilham em suas redes sociais. Por outro lado, relata que o uso da tecnologia das redes sociais ajuda constantemente os profissionais de saúde através de mensagens por aplicativo a resolverem os casos de seus pacientes no quesito de troca de informações referentes ao tratamento. Garrafa (2005) relata que os bioeticistas estadunidenses, vêm trabalhando a bioética a partir de uma base conceitual estabelecida sobre princípios preestabelecidos. A teoria principialista, universalizada por Beauchamp e Childress, tomou como parâmetro quatro princípios básicos, sendo autonomia, beneficência, 


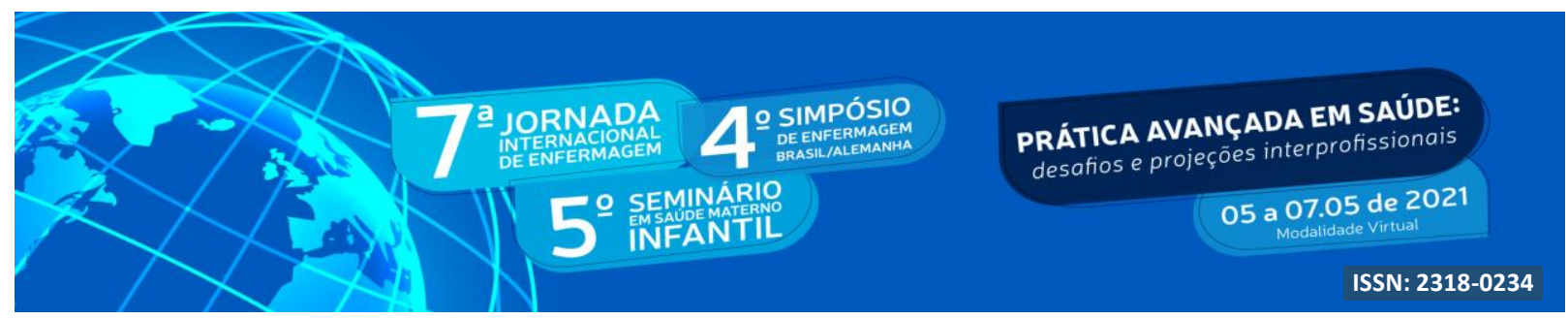

não maleficência e justiça, os quais seriam uma espécie de instrumento simplificado para uma análise prática dos conflitos que ocorrem no campo bioético.

Limaye et al. (2020) afirmam a importância das redes sociais para aproximar e informar as pessoas principalmente no período de confinamento por causa da Covid -19, porém alerta que o uso de forma excessiva e muitas vezes o compartilhamento de informações errôneas, causam pânico e pavor na população.

É de entendimento geral que fatores como a comunicação, a disseminação e a troca de informações são indispensáveis atualmente. Na área da saúde, a comunicação entre os profissionais se faz fundamental, pois processos comunicativos estão constantemente inseridos em suas atividades cotidianas (DE CAMARGO; ITO, 2012). Porém este caso reacende a discussão sobre o acesso a informações privilegiadas que alguns estudos, instituições, organizações ou pessoas podem obter informações de outros membros da sociedade. Nos códigos de ética profissional, os itens se referem, os itens que representam à relação profissional-paciente normalmente trazem o sigilo de informações como um direito do paciente; garantir este direito é um dever do profissional. Em algumas situações no qual o profissional de saúde se sinta intimidado a revelar um segredo, como nos casos das relações de subordinação trabalhista, o respeito ao sigilo também pode ser recorrido como um direito deste profissional (MARTORELL; NASCIMENTO; GARRAFA, 2015; SALES-PERES, 2008).

Os profissionais acessam informações pessoais confidenciais de pacientes para entender seus problemas de saúde, e tais informações são de fato confidenciais, determinando que aqueles que a possuem têm a responsabilidade de protegê-las de divulgação para alheios (BUDIMIR et al., 2013). A contínua busca por melhorias e disponibilização de novos produtos por parte das instituições levaram vários setores a investir em sistemas de informação e tecnologia na área da saúde. Há uma crescente popularização do uso e redes sociais entre os profissionais da área da saúde, que passam a usar tal ferramenta publicando na internet informações sobre suas rotinas profissionais, acabando em muitas situações, expondo situações inadequadas, se arriscando a quebrar o sigilo e a confidencialidade que devem a seus pacientes (MARTORELL; NASCIMENTO; GARRAFA, 2015).

$\mathrm{Na}$ área da odontologia e da medicina estética é frequente o uso de imagens de partes 


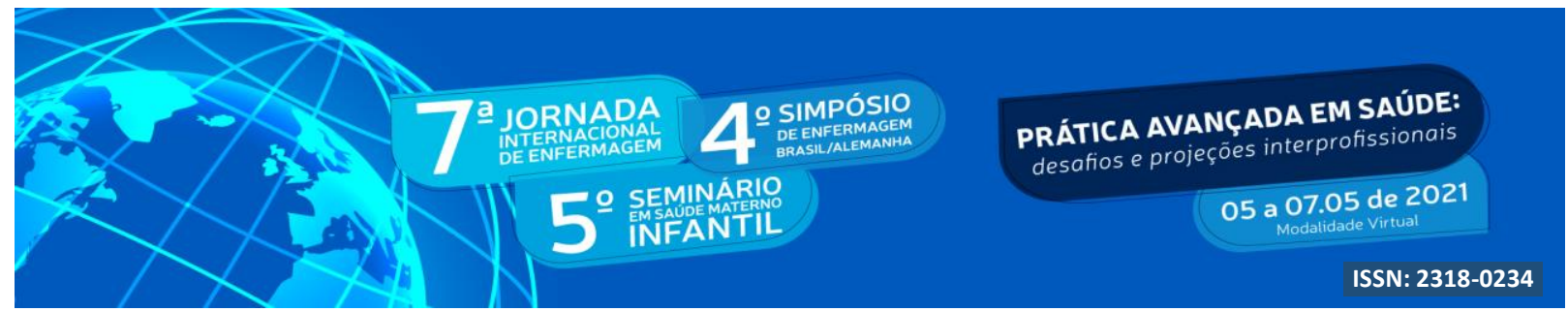

do corpo que não identificam o paciente em questão, porém ainda assim pode haver algum constrangimento. Mesmo que o público em geral não possa reconhecer o sujeito esse poderá se sentir contrangido com certa exposição de seu tratamento ou condição. Um sorriso com um antes ou depois de um tratamento odontológico, um seio antes ou depois de uma cirurgia plástica. Mesmo que seja partes do corpo da pessoa, a divulgação de imagens sem o consentimento pode remeter o indivíduo a um sofrimento psíquico, apesar contemporaneidade, as pessoas queiram e busquem a exposição (NASCIMENTO, 2016).

O Conselho Federal de Medicina (CFM), no Brasil, a Resolução N ${ }^{\circ}$ 1931/2009 (Código de Ética Médica) IV, capítulo IX artigo 73, trata a questão do sigilo, como forma de garantir a privacidade do paciente. Decreta que: "É vedado ao médico: Revelar fato de que tenha conhecimento em virtude do exercício de sua profissão, salvo por motivo justo, dever legal ou consentimento, por escrito, do paciente". Porém a resolução 196/2019 veio para atualizar alguns pontos importantes referentes ao Código de Ética da Odontologia, trazendo mais liberdade para os dentistas divulgarem seus tratamentos e serviços, inclusive podendo postar imagens de antes e depois dos tratamentos (NAVES, 2010; MARTORELL; DO PRADO; FINKLER, 2019).

Falar sobre o consentimento do paciente e também refletir sobre o constrangimento que o paciente pode sentir ao não querer autorizar essa divulgação. A exposição indiscriminada e acrítica de casos clínicos relacionados à prática odontológica em mídias sociais é uma preocupação crescente no que tange aos aspectos éticos e legais que regem a profissão, Felter et al. (2017) relatam a importância das mídias na educação continuada dos profissionais da área da saúde, porém ressaltar que a mídia serve também para a propagação de ideias equivocadas do ponto de vista da ciência , tornando-se, assim, local de exposição reprovável de conteúdos e ou pessoas que viola a privacidade, o sigilo e a confidencialidade de dados de pacientes.

Costa et al. (2020) avalia o comportamento dos dentistas no uso das imagens dos pacientes no planejamento e discussão dos casos com outros colegas. Embora estes exames são suma de importância na clínica e para pesquisa, o autor conclui que os profissionais devem solicitar um consentimento informado para compartilhar em suas redes sociais ou com outros colegas de forma privada as imagens de seus pacientes e principalmente aquelas que 


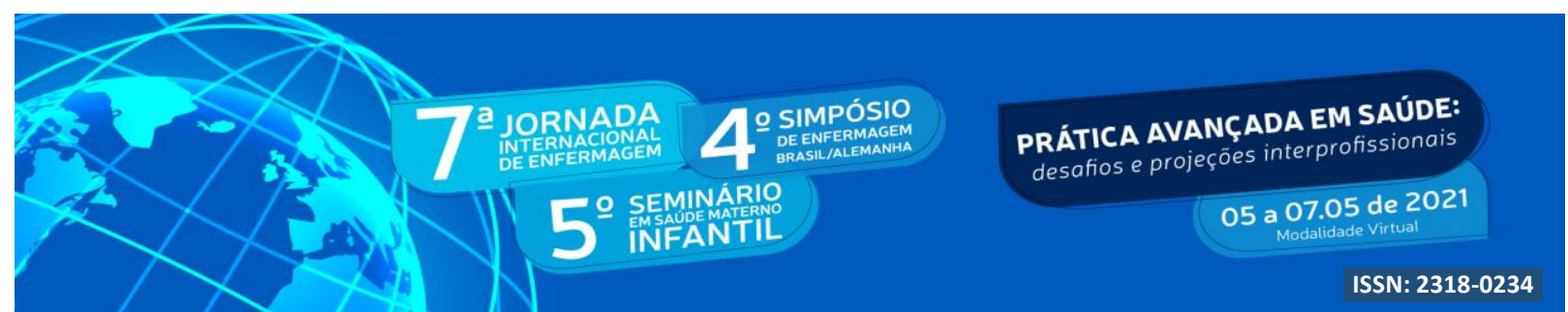

revelem identidade.

Sob a perspectiva da "ética da proteção" e da "bioética de intervenção", Fagundes e colaboradores em 2007 analisa a influência das peças publicitárias de medicamentos veiculadas à classe médica na prescrição de medicamentos. Estuda, ainda, a qualidade das informações nas propagandas de medicamentos de venda sob prescrição, antes e depois da Resolução da Diretoria Colegiada da Anvisa - RDC 102/2000, que regulamenta a propaganda de medicamentos no país, bem como discute o papel regulador do Estado na área. A presente pesquisa constatou que, embora algumas mudanças legais tenham acontecido nos últimos anos, continuam ocorrendo irregularidades, em desacordo com a legislação vigente, influenciando a prescrição e o consumo de medicamentos e, principalmente, induzindo as pessoas à automedicação.

\section{CONCLUSÃO}

Apesar de enfrentarmos um crescimento constante do uso das tecnologias e redes sociais na área da saúde, ainda são escassos os estudos que se dispõem a fazer uma reflexão aprofundada sobre a privacidade, confidencialidade e os aspectos bioéticos relacionados a essa temática. Os aspectos bioéticos do uso de informações dos pacientes e sua divulgação deve ser amplamente discutido entre profissionais da área da saúde no intuito de garantir o sigilo dos dados por eles acessados, a privacidade dos pacientes e a qualidade das informações disponibilizadas ao publico geral. Além disso, é necessário informar a população de seus direitos enquanto pacientes no que tange a divulgação de seus dados, da obrigatoriedade da sua anuência por escrito para que qualquer informação ou imagem seja divulgada e principalmente da sua autonomia em consentir ou não, sendo sua decisão soberana e isenta de qualquer tipo de constrangimento em qualquer uma dessas etapas.

\section{REFERÊNCIAS}

ANTHEUNIS, M.L.; TATES, K.; NIEBOER, T.E. Patients' and health professionals' use of social media in health care: motives, barriers and expectations. Patient education and counseling, v. 92, n. 3, p. 426-431, 2013. [citado 15 de mai 2020]. Disponível: https://www.sciencedirect.com/science/article/abs/pii/S0738399113002656 


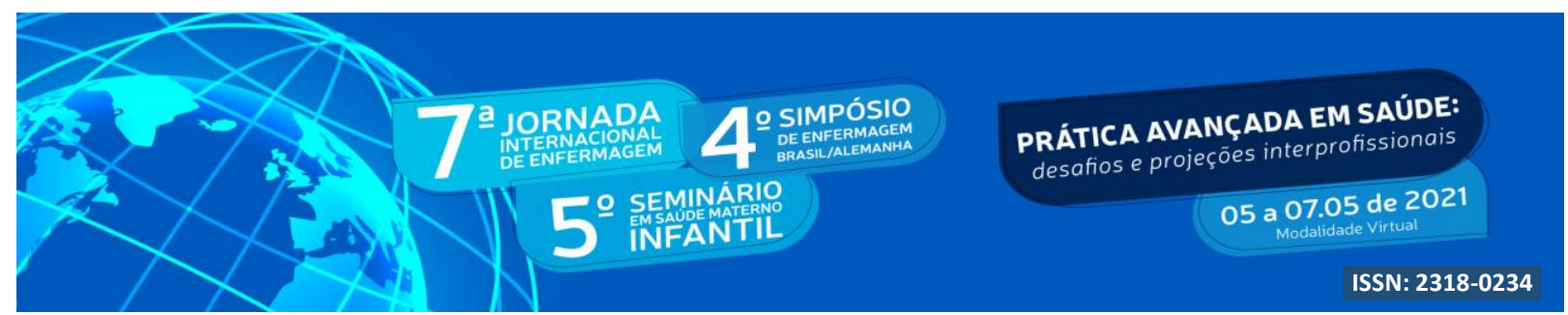

BUDIMIR, V. et al. Knowledge, attitudes, and awareness of Croatian dentists concerning patients' confidentiality and privacy. Journal of dental education, v. 77, n. 3, p. 370-376, 2013. [citado em 25 de mai 2020]. Disponível:

https://onlinelibrary.wiley.com/doi/epdf/10.1002/j.0022-0337.2013.77.3.tb05481.x

CAMPOS V.F.; SILVA J.M.; SILVA J.J. Comunicação em cuidados paliativos: equipe, paciente e família. Rev. bioética. (Impr.). v.27, n.4, p.711-718, 2019 [citado 10 de jun 2020). Disponível: https://www.scielo.br/scielo.php?script=sci_arttext\&pid=S1983$\underline{80422019000400711 \& \text { lang }=\mathrm{pt}}$

DE CAMARGO, A.L; ITO, M. Utilização das tecnologias de informação e comunicação na área da saúde: uso das redes sociais pelos médicos. Journal of Health Informatics, v. 4, n. 4, 2012. [citado 04 jun 2020] Disponível: http://www.jhi-sbis.saude.ws/ojs-jhi/index.php/jhisbis/article/view/220/144

FAGUNDES, M.J.D. et al. Análise bioética da propaganda e publicidade de medicamentos. Ciência \& Saúde Coletiva, v. 12, p. 221-229, 2007. [citado 28 de mar 2020]. Disponível: https://www.scielosp.org/article/csc/2007.v12n1/221-229/pt/

FELTER, M.et al. A violação dos aspectos éticos e legais de uma rede social profissional odontológica. RBOL-Revista Brasileira de Odontologia Legal, v. 4, n. 3, 2017. [citado 28 de mar 2020]. Disponível:

http://portalabol.com.br/rbol/index.php/RBOL/article/view/127/145

GARRAFA, V. Reflexões bioéticas sobre ciência, saúde e cidadania. Revista Bioética, v. 7 , n. 1, 2009. [citado 10 de jun 2020] Disponível:

http://revistabioetica.cfm.org.br/index.php/revista bioetica/article/viewFile/287/426

GARRAFA, Volnei. Da bioética de princípios a uma bioética interventiva. Revista Bioética, v.13, n.1, 2005. [citado 30 de jan 2021] Disponível:

https://revistabioetica.cfm.org.br/index.php/revista_bioetica/article/view/97/102

LIMA, A.I.C.; CRUZ, R; DA SILVA, R. Análise dos perfis de clínicas odontológicas e de cirurgiões-dentistas em duas redes sociais quanto aos aspectos éticos, de propaganda e publicidade. RBOL-Revista Brasileira de Odontologia Legal, v. 3, n. 2, 2016. [citado 10 de mai 2020] Disponível: http://portalabol.com.br/rbol/index.php/RBOL/article/view/72/92

LIMA, C.V.C; BIASOLI, L.F. Interfaces, gaps and challenges between bioethics and oncology. Revista Bioética, v. 26, n. 3, p. 451-462, 2018. [citado 5 de jun 2020] Disponível: https://www.scielo.br/scielo.php?pid=S1983-80422018000300451\&script=sci_arttext

LIMAYE, R.J.et al. Building trust while influencing online COVID-19 content in the social media world. The Lancet Digital Health, v. 2, n. 6, p. e277-e278, 2020. [citado 28 de mai 2020]. Disponível: https://www.thelancet.com/action/showPdf?pii=S2589$\underline{7500 \% 2820 \% 2930084-4}$ 


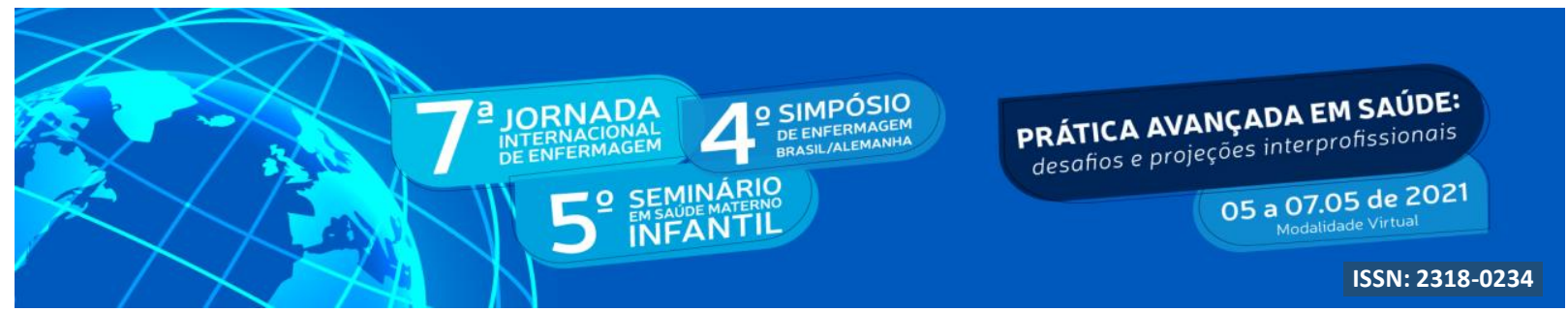

MARTORELL, L.B.; NASCIMENTO, W.F.; GARRAFA, V. Redes sociais, privacidade, confidencialidade e ética: a exposição de imagens de pacientes no facebook. InterfaceComunicação, Saúde, Educação, v. 20, p. 13-23, 2015. [citado 28 mai 2020]. Disponível: https://www.scielosp.org/article/icse/2016.v20n56/13-23/

MARTORELL, L.B. Uso de mídias sociais: um caso de urgência e emergência para profissionais da saúde. RBOL-Revista Brasileira de Odontologia Legal, v. 4, n. 1, 2017. [citado 10 jun 2020]. Disponível:

http://portalabol.com.br/rbol/index.php/RBOL/article/view/130/129

MARTORELL, L.B.; DO PRADO, M.M; FINKLER, M. Paradoxos da Resolução CFO n. 196/2019:"Eu tô te explicando, pra te confundir". RBOL-Revista Brasileira de Odontologia Legal, v. 6, n. 1, 2019. [citado 06 de jun 2020]. Disponível:

http://portalabol.com.br/rbol/index.php/RBOL/article/view/252/200

MCKEE, R. Ethical issues in using social media for health and health care research. Health Policy, v. 110, n. 2-3, p. 298-301, 2013. [citado 15 mai 2020]. Disponível:

https://www.sciencedirect.com/science/article/abs/pii/S0168851013000468

NASCIMENTO, D.B. Análise bioética da utilização de imagens de pacientes em mídias sociais eletrônicas. 2016. xiii, 90 f., il. Dissertação (Mestrado em Bioética)—Universidade de Brasília, Brasília, 2016.

NORMAN, I.D.; AIKINS, M.K.; BINKA, F.N. Ethics and electronic health information technology: challenges for evidence-based medicine and the physician-patient relationship. Ghana medical journal, v. 45, n. 3, p. 115, 2011. 2011 [citado 10 de jun 2020]. Disponível: https://www.ncbi.nlm.nih.gov/pmc/articles/PMC3266146/

POSSOLLI, G.E.; DO NASCIMENTO, G.L.; DA SILVA, J.O.M. A utilização do Facebook no contexto acadêmico: o perfil de utilização e as contribuições pedagógicas e para educação em saúde. RENOTE-Revista Novas Tecnologias na Educação, v. 13, n. 1, 2015. [citado 20 de mai 2020]. Disponível: https://www.seer.ufrgs.br/renote/article/viewFile/57586/34564

SALES-PERES, S.H.C. et al. Sigilo profissional e valores éticos. RFO UPF, v. 13, n. 1, p. 713, 2008. [citado 15 jun 2020]. Disponível: http://seer.upf.br/index.php/rfo/article/view/583

THOMPSON, L.A. et al. The intersection of online social networking with medical professionalism. Journal of general internal medicine, v. 23, n. 7, p. 954-957, 2008. [citado 10 de jun 2020]. Disponível: https://link.springer.com/article/10.1007\%252Fs11606-008$\underline{0538-8}$ 